\title{
The current status and challenges in the diagnosis and treatment of rheumatoid arthritis in China: An annual report of 2019
}

Xinping Tian, Mengtao Li, Xiaofeng Zeng*

Department of Rheumatology, Peking Union Medical College Hospital (PUMCH). Chinese Academy of Medical Sciences, National Clinical Research Center for Dermatologic and Immunologic Diseases (NCRC-DID), Key Laboratory of Rheumatology and Clinical Immunology, Ministry of Education, Chinese Rheumatism Data Center (CRDC), Beijing 100730, China

Received February 1, 2021 accepted February 23, 2021

\section{Introduction}

Rheumatoid arthritis (RA) is a chronic and highly destructive autoimmune disease characterized by symmetrical joint pain and swelling. The disease course is typically progressive with intermittent remission. If patients are not given the appropriate treatment, a significant number of them would develop joint deformities and some may eventually even become disabled. Among systemic rheumatic diseases, RA is the most common in China and has been called "a malignancy without death" by some Chinese individuals. The disease not only causes physical impairment and mental stress, but also leads to huge financial burdens to patients, their families, and society.

In July 2019, under the leadership of the China Rheumatism Data Center (CRDC), we drafted the first annual report of RA in China. In this report, we comprehensively reviewed the current status, problems, challenges, and opportunities physicians are faced with in the diagnosis and management of RA in China. We also developed a prospective of the development of Chinese rheumatology in the future. The goal of this report is to provide scientific evidence for healthcare administrative agencies at all levels to formulate the relevant policies and properly allocate resources for RA diagnosis and management. This report has also provided guidelines for Chinese rheumatologists and other physicians to improve the quality of care for RA and practice standardized management and prevention.

\section{The Epidemiologic Assessment and Clinical} Characteristics of RA Patients in China

\footnotetext{
Address for correspondence:

*Xiaofeng Zeng, Professor, Department of Rheumatology, Peking Union Medical College Hospital (PUMCH), Chinese Academy of Medical Sciences, National Clinical Research Center for Dermatologic and Immunologic Diseases (NCRC-DID), Key Laboratory of Rheumatology and Clinical Immunology, Ministry of Education, Chinese Rheumatism Data Center (CRDC), Beijing 100730, China.

E-mail: xiaofeng.zeng@cstar.org.cn
}

There have been limited epidemiological studies of RA in China till date. Most studies were retrospective or based on in-patient case analysis. As a result, these study results may not reflect the actually prevailing situation regarding RA in China. In November 2016, the Chinese Registry of Rheumatoid Arthritis (CREDIT) program was established by CRDC, and it has prospectively registered RA patients from over 300 hospitals across the country. In this registry, demographic data, clinical manifestations, laboratory tests, diagnosis, treatment regimens, and prognosis have been collected and recorded. Over 50,000 RA patients have been registered in the past 4 years. This registry has provided the first-hand data for physicians and public to understand the disease in more detail.

\section{The Epidemiological Characteristics of Chinese RA Patients}

Compared with international reports on RA, our data have shown that the RA population in China is the biggest. It is estimated that there are approximately 5 million RA patients in China. The average age of disease onset is 45 years and it peaks around age 50 . The disease is more prevalent in females than males with a ratio of $4: 1 .^{[1]} \mathrm{RA}$ is more prevalent in northern China and in the southern part of the country. There is no significant difference between the urban and rural areas. ${ }^{[1]}$ In addition, Chinese RA patients have a longer disease course and more severe disease with higher disease activity. Comorbidities and RA complications also occur more frequently in Chinese patients. 
The data from CREDIT showed a mean 2-year delay in establishment of the diagnosis after the onset of symptoms. This indicated that a majority of Chinese RA patients were not diagnosed at an early stage. From a cross-sectional study data in CREDIT, we could ascertain that $34.82 \%$ Chinese RA patients were found to have moderate disease activity and $47.18 \%$ to have high disease activity. In other words, most Chinese RA patients had moderate to severe disease activities. Based on the data from CREDIT, RA patients also had interstitial lung disease, fragile fracture, cardiovascular diseases, and malignancy, to the extent of $10.32 \%, 1.97 \%$, $3.04 \%$, and $0.83 \%$ respectively. These complications would make the management of RA even more difficult.

\section{RA Prevalence is Increasing and Patients Tend to be Younger at Disease Onset}

In 1983 , RA prevalence was estimated to be $0.30 \%$ in China ${ }^{[2]}$; this rate increased to $0.42 \%$ as reported by Zeng X, et al..$^{[3]}$ in 2013. In contrast, global RA prevalence was reported to be $0.24 \%$ during the same time period. ${ }^{[4]}$ It is expected that the rate of RA in China would increase even more when earlier diagnoses of RA are made over time.

The data from CREDIT also showed an average age of $45.47 \pm 14.4$ years at disease onset in 2019. In the same year, the average age of RA patients was found to be 51 years. Patients in this age are the "backbone" of their families and communities; thus, this population with RA as a main workforce may have serious impacts economically. In contrast, globally the average age at disease onset was 55.4 years, and the average age of the RA patient population was $60-64$ years in $2019 .{ }^{[5]} \mathrm{A}$ majority of patients at this age are retired from their workforce. Therefore, after comparison with the global data, the fact is ascertained that RA causes a much heavier financial burden on Chinese families and the entire Chinese society.

\section{RA Prognosis is Worse in China than Internationally Reported Levels}

Chinese RA patients may have multiple extraarticular organ and system involvement. Furthermore, chronic inflammation of RA and adverse reactions caused by long-term drug treatment put patients at high risk for more complications. According to the data from CREDIT, $10.32 \%$ of RA patients had interstitial lung disease, compared with $3.34 \%$ as reported internationally. RA patients with interstitial lung disease had a much worse prognosis compared with those without. Chinese RA patients $(76.14 \%)$ meanwhile had 3-4 clinical and laboratory features for poor prognosis, and only $1.26 \%$ Chinese RA patients displayed no feature for poor prognosis. In addition, such complications as coronary heart disease, stroke, fragile fracture, and malignancy occurred in $1.88 \%, 0.92 \%, 1.70 \%$, and $0.60 \%$ respectively in Chinese RA patients, based on an analysis in 2017. Furthermore, these complications increased to $2.05 \%, 0.99 \%, 1.97 \%$, and $0.83 \%$, respectively, in 2019 . It may be expected that the RA population with severe disease would increase as patients lived longer.

\section{RA-related Financial Burden is Much Heavier in China than in Other Countries}

RA is a life-long disease with a high disability rate. According to the Second China National Population Survey on Disability, which was conducted in 2006, joint diseases contributed to physical disability as a major cause in China, with RA at the top of the list. If disability is defined as a joint deformity with functional impairment, the disability rate would be $43.48 \%$ in Chinese RA patients with disease duration of 5-10 years. The disability rate would increase to $61.25 \%$ if disease duration lasted over 15 years. ${ }^{[6]}$ If disability is defined based on the disability criteria elucidated Beijing Municipal government, complete disability rate would be $30-40 \%$ in Chinese RA patients with disease duration of over 10 years. In contrast, according to an international report, $80 \%$ of RA patients became partially disabled in patients whose disease lasted for 12 years, and only $16 \%$ of RA patients became completely disabled. ${ }^{[7]}$

Costs associated with pharmacologic treatment of RA and its related disability have caused heavy economic burden to the patients' families and the society. A global survey showed that RA associated burden was ranked number one among rheumatic diseases in 2015. ${ }^{[8]}$ Globally, the drug cost for treating RA was US $\$ 21.4$ billion in $2015 .^{\left[{ }^{[9]}\right.}$ In the United States, the direct medical cost for treating RA was US $\$ 12,509$ per patient each year. The medical cost for 6 months was US\$728 in Japan. ${ }^{[10]}$ In China, the cost for management of RA patients was US $\$ 12$ million each year. If we were to take the Disability Adjusted Life Years (DALYs) into consideration, the overall economic cost would increase to US $\$ 0.15$ billion each year and the average economic cost would be US $\$ 2245$ per patient each year. ${ }^{[11]}$

\section{Challenges In RA Management In China}

\section{Inadequate Public Awareness of the Disease}

Due to the limited availability of propagation materials on scientific knowledge of RA, public awareness of RA is very low in China. Studies have shown that $35.80 \%$ of Chinese RA patients obtained some knowledge of the disease from 
their relatives, $25.30 \%$ from drug advertisements, and $18.80 \%$ from the internet. Over $50 \%$ of the public could not differentiate between the two concepts of "RA" and "rheumatic arthritis (rheumatic fever)." Approximately $90 \%$ of the general public did not know the appropriate medical department or physicians to seek to avail medical care. ${ }^{[12]}$ As a result, a diagnosis of RA was not made timely. ${ }^{[13]}$ Even some medical care professionals are known to have very limited knowledge of RA.

Furthermore, RA patients were unaware of the importance of the standard medical treatment for better long-term prognosis. Some patients were addicted to "folk medicine," "secret recipes passed down from generations to generations," and "radical therapy"; therefore, they did not receive the standard medical care for RA, and consequently missed the opportunity to gain timely treatment. Some patients only received standard treatment when their disease progressed to the advanced stage with severe deformity. All these factors were closely related to the high disability rate in Chinese RA patients. Most Chinese RA patients did not understand that RA needs to be treated in a "tight-control" approach for a prolonged period of time, and they did not know the importance of "treat-to-target" to gain better prognosis with persistent remission and less disability. As a result, most patients often self-discontinued medications upon disease remission. Consequently, these instances led to more severe, refractory, and advanced disease in China.

Rheumatology is the youngest subspecialty of internal medicine in China. Many hospitals, primary care hospitals in particular, do not have a free-standing department of rheumatology, and therefore, the knowledge of rheumatic diseases is not widely available. Some medical professionals, especially primary care physicians, have very limited knowledge of the clinical features, diagnosis, assessment, pharmacologic treatment, and prognosis of RA. Some primary care professionals also could not differentiate between RA and rheumatic arthritis related to rheumatic fever. Further, because of the lack of access to validated diagnostic laboratories and tests, RA was often misdiagnosed in primary care settings. Numerous patients, as a result of being unable to obtain an early diagnosis, missed the opportunity for timely treatment.

In the past, most rheumatologists did not obtain standardized rheumatology fellowship training. Currently, however, Chinese rheumatologists have different levels of knowledge, skills, and experiences in relation to RA. Some rheumatologists under-recognize the importance of a tight-control strategy and treat-to-target principle in their daily management of RA. This is reflected in two aspects. One aspect is that some rheumatologists did not treat patients using standardized therapy and did not even adjust medications based on disease activity. The other aspect is that some rheumatologists did not follow-up or monitor patients regularly. These aspects have led to a low disease remission rate in China. Data from CREDIT has shown that only $9.26 \%$ of RA patients were able to follow-up with their physicians regularly for 1 year or more.

\section{Low Rate of Early Diagnosis}

The misdiagnosis and under-diagnosis of RA in China is relatively common, particularly in primary care settings. Although more RA patients had an early diagnosis over time, the average time from symptom onset to diagnosis confirmation was 2.5 years, according to the data from the CREDIT registry. The best treatment window of opportunity is within 6-12 months after the disease onset. As a result of the delayed early diagnosis, more patients had moderate to high disease activity when they first sought medical care from their rheumatologists. Therefore, there were more refractory cases of RA and lower remission rates in China when compared with the internationally reported data.

\section{Low Rate of Using Standardized Treatment}

Studies have shown that pharmacologic prescriptions for RA were categorized as "two higher and two lower" phenomena. There were higher rates of prescriptions for NSAIDs and glucocorticoids, whereas disease modify anti-rheumatic drugs (DMARDs) such as methotrexate, biologics, and small molecule target drugs were prescribed less frequently when compared with the international report. Based on the CREDIT registry data, $69.70 \%$ of Chinese RA patients were treated with glucocorticoids for longer than 6 months and $11.30 \%$ were treated with glucocorticoid monotherapy. ${ }^{[14]}$ Methotrexate has been regarded as the anchor drug for the treatment of RA. Most RA patients should be treated with methotrexate unless they are contraindicated or intolerant to the drug. However, only $51.60 \%$ of Chinese RA patients were treated with methotrexate, whereas $83 \%$ were reportedly treated in European countries and the United States. ${ }^{[15]}$ The prescription rates of other first-line drugs (sulfasalazine and hydroxychloroquine) were $8.24 \%$ and $0.70 \%$, respectively; these rates are much lower than those reported globally. Another evident phenomenon was a shorter duration of DMARDs use. For example, $85.25 \%$ of patients were treated with DMARDs for less than 6 months.

\section{Lack of Efficient Chronic Disease Management System}

Over the years, partly due to the healthcare system, Chinese RA patients have not been used to a long-term regular follow- 
up with physicians yet, and thus have a low compliance rate to treatment. The National Nursing Cooperative Group for Chronic Rheumatic Disease Management developed the consensus for chronic rheumatic disease management in 2014, but there have been little evidence-based standards and guidelines for the management of chronic rheumatic diseases; particularly, there has been no specific model for chronic disease management available in China. Rheumatologists or physicians have often had no guidance or policy to follow or habit to monitor patients regularly.

\section{Severe Shortage of Rheumatologists}

In China, rheumatology is a relatively young specialty and the development of rheumatology has much lagged behind other subspecialties of internal medicine. As a result, rheumatologists are in severe shortage in relation to other subspecialties. In 2007, the Rheumatology and Immunology Physicians Committee of the Chinese Medical Doctors Association conducted a national survey on the rheumatology workforce in the country. ${ }^{[16]}$ The survey included 701 secondary and tertiary hospitals around the country. A total of 738 departments participated in the survey. The results showed that 2216 rheumatologists were involved in the diagnosis and treatment of rheumatic diseases. A total of 1891 (85.30\%) rheumatologists worked in the tertiary hospitals while $323(14.60 \%)$ worked in the secondary hospitals. A total of 1267 rheumatologists worked in the free-standing department of rheumatology, only accounting for $57.20 \%$ of all the rheumatologists in the country. Rheumatologists accounted for $0.064 \%$ of all registered clinicians in the country in 2017 . This indicated that every 10 million people in China shared 16.7 rheumatologists. This was much less than the 166.7 rheumatologists for every 10 million people in the United States. ${ }^{[17]}$ A majority of Chinese rheumatologists were aged between 31 and 45 years and had less than 10 years' experiences in medical practice. Most Chinese rheumatologists had medical college education or a master's degree. The survey also revealed that Chinese rheumatologists worked in large cities, such as Beijing, Shanghai, and in the coastal areas like Shandong, Jiangsu, and Guangdong provinces. As of October 1, 2007, 1035 (46.70\%) rheumatologists had never trained in a rheumatology fellowship program in China, $105(4.70 \%)$ rheumatologists had trained abroad, and only $56(2.50 \%)$ rheumatologists had trained in a rheumatology fellowship program domestically and studied abroad. The above data show that the quality and quantity of Chinese rheumatologists could not meet the needs of Chinese patients with rheumatic diseases. Rheumatology is still a very small and weak subspecialty in the country. Many hospitals throughout the country do not have an independent department of rheumatology. Moreover, the distribution of rheumatologists is not balanced across the country. Due to the serious shortage of rheumatologists, further development of rheumatology, including increase of rheumatologist training sites, and enlargement of the rheumatology workforce, is a critical requirement in the future.

\section{Research and Achievements on RA in China}

\section{Scientific Research of RA by Rheumatologists}

Recently, Chinese rheumatologists have dedicated themselves to the basic and clinical study of RA. Some examples of their research results are listed in the following report.

\section{Basic science research}

Most studies were focused on the potential pathogenesis and treatment targets of RA. Recently, Chinese scientists have discovered that $C D 109$ is involved in the pathogenesis of RA, suggesting that it could be a potential therapeutic target for RA. ${ }^{[18]}$ One study has shown that methylation of the PARP9 gene plays a role in the pathogenesis of RA. ${ }^{[19]}$ In addition, Sun et al. have found that $B$ cells are able to inhibit new bone formation by producing multiple osteoblast inhibitors. ${ }^{[20]}$

Feng et al. ${ }^{[21]}$ developed a fluorescence-labeled hypochlorous acid Probe-2, which can predict the patient's therapeutic response to methotrexate. This probe could be used as a tool for the diagnosis and therapeutic monitoring of several inflammatory diseases, including RA.

Wang et al. ${ }^{[2]}$ found that lung involvement in RA patients is associated with a specific genetic background and chronic airway and alveolar damage. This finding has provided evidence for the potential development of drugs for RArelated lung diseases. Another finding is that correction of the imbalanced microbiota in the disease back to a healthy composition could be a potential therapy for bone and joint damage in RA. ${ }^{[23]}$ Some related trials are underway. For example, trials of melatonin as an adjunctive treatment for $\mathrm{RA}^{[24]}$ and nanosized drugs for the treatment of RA are ongoing. ${ }^{[25]}$

\section{Clinical research}

In the past 10 years, Chinese rheumatologists have made substantial achievements in clinical research. Two most important examples of such achievements include establishment of a nation-wide registry and development of a model to enhance the treat-to-target strategy in the country. It is expected that these accomplishments could further 
improve the diagnosis and treatment of RA in China.

\section{Establishment of a Large Nation-wide Registry for RA}

The RA nation-wide data registry has become an important tool for conducting large-scale and high-quality real-world studies. The disease registry has become an important supplement for randomized controlled clinical trials. The nation-wide registry has enabled us to understand the epidemiological trends, monitor therapy, and identify factors related to poor prognosis. In Europe, an inter-country registry has been used to carry out collaborative study of RA. A wellknown international RA registry is CORRONA, developed in the United States in 2001. A total of 51,000 RA patients were registered and more than 0.4 million visits were included in the registry. More than 140 research articles were published based on the data from the registry. CORRONA is regarded as the "gold standard" for the RA registry. The British Society for Rheumatology Biologics Register (BSRBR) was established in 2001, and this registry has registered approximately 5000 RA patients. Included in this number are more than $80 \%$ of the RA patients who were treated with biologics. The goal of the registry is to evaluate the long-term safety of biologics in RA patients. This registry has provided firsthand data to understand the efficacy and safety of biologics in Britain. The Optimizing Patient Outcomes in Australian Rheumatology (OPAL), which was developed in 2009 , aims to enhance the management of patients with chronic diseases. The Institute of Rheumatology Rheumatoid Arthritis Cohort (IORRA) registry was established in Japan in 2000. This database has collected information about genetics, comorbidities, drugs, healthcare insurances, and drug economics. Over 100 articles have been published from data from the IORRA registry. ${ }^{[26]}$

Having learned from the experiences of these well-established registries, the first nation-wide registry for RA patients was set up in China in November 2016. This registry was based on the technical platform of CRDC in China and was developed under the auspices of the CREDIT program. The registry was established to initiate RA patient cohort development in China. It has enabled Chinese rheumatologists to understand the status of RA and conduct high-quality and state-of-the-art studies.

\section{Enhancing Treat-to-target Strategy by Setting up the CREDIT Program}

The CREDIT program was initiated in November 2016. It is the first nation-wide multicenter online registry. The goal of the CREDIT program is to enhance "treat-to-target" principle by implementing the "tight-control" policy across the country.
The program combines online registry, a patient follow-up reminding system, and patient education with physician training courses. It also integrates with the "one city, one department of rheumatology, one medical center for rheumatic diseases" program to facilitate academic development in relation to rheumatology across the country. This program pays special attention to patient care. A "caring for RA patients around you" App was built to spread knowledge about the disease, to introduce medications used in the treatment of the disease, and to prompt the concept of treat-to-target. This App also serves as a reminder for follow-up visits.

This model has turned out to be a great success. As of October 31, 2019, 1484 rheumatologists from 417 hospitals in 31 provinces participated in the program and registered their RA patients into the registry; moreover, 53,559 RA patients with complete demographic data, clinical presentations and laboratory test results, disease activity assessment and complications, and prognosis were registered. To our knowledge, this is the biggest RA patient cohort in the world, to date. This registry has provided the infrastructure for state-of-the-art clinical studies in China. As of October 2019, 4 articles were published based on data from the CREDIT program. The CREDIT program has opened a window for rheumatologists around the world to understand the progress of management of RA in China.

\section{Rapid and High-quality Development of Chinese Rheumatology}

Rheumatologists are in serious shortage in China, and the levels of patient care are not adequate to provide standardized care for RA patients. In addition, rheumatologists are not evenly distributed across the country. To speed up the development of rheumatology in China, it would be essential to build a high-quality workforce and set up a national network of rheumatologists. These would meet the needs of Chinese patients, and enable them to be transferred between primary, secondary, and tertiary hospitals around the whole country.

\section{Development of a nation-wide collaboration network}

In order to implement the "Plan for Healthy China 2030,", the General Office of the State Council has issued the "Guidance and Suggestions for Prompting Medical Care Alliance Development." The National Health Commission of the People's Republic of China has issued "Guidance for Pilot Medical Care Alliance Development." The Chinese Rheumatology Center Alliance (CRCA) was developed in Beijing in November 2017 under the joint leadership of the Department of Rheumatology of Peking Union Medical College Hospital (PUMCH) and CRDC. The goals of this organization 
are to improve the balance of health care resources across the country, to explore the model of development of health care alliance in rheumatology, and to solve the problems including such as shortage of rheumatologists and low-level of health care around the country. The CRCA is composed of health care institutions of in the country and rheumatologyassociated organizations, including research institutes and pharmaceutical companies. In addition, the CRCA enables adjustment and optimization of health care resources across the country and facilitates the standardized treatment of RA in the primary care setting. This would move the hierarchical health care system development forward, improve the overall effectiveness of the health care system, and meet the medical care needs of Chinese patients with rheumatic diseases.

\section{Prompting the "one city, one department of rheumatology, one medical center for rheumatic diseases" program}

In 2015, under the leadership of the Chinese Rheumatology Association (CRA), and with the assistance of the Rheumatology and Immunology Physicians Committee of Chinese Medical Doctors Association, a program with the goal of "One city, one department of rheumatology, one medical center for rheumatic diseases" was established. In this program, rheumatic disease centers are developed at the regional level. These centers will exert their impacts on their affiliated regions to facilitate the development of rheumatic disease departments or centers at different geographic-levels. This program will speed up the formation of more free-standing rheumatic disease departments across the country. This may partially alleviate the shortage of rheumatologists. Since the initiation of this program, rheumatology associations have been formed in all provinces of the country. Many provinces have also established associations for young rheumatologists to attract young physicians to the field. This has contributed greatly to the development of the future rheumatology workforce. The number of departments of rheumatology has doubled, and the number of rheumatologists increased to more than 12,000 in 2019. There were 1256 independent departments of rheumatology across the country in 2019. Compared with 2018, 76 more departments were added with an increase of $6.40 \%$ in 2019 ; however, this number is still behind the $7.50 \%$ increase in other subspecialties of internal medicine. Eleven provinces have achieved the goal of "One city, one department of rheumatology, one medical center for rheumatic diseases." The number of rheumatologists with different levels of academic degrees has increased, along with a dramatic increase in rheumatologists with a doctoral degree.

\author{
Formulating the guidelines for RA diagnosis and \\ treatment
}

The CRA published the first guideline for RA diagnosis and treatment in 2004[27] and revised it in 2010. ${ }^{[28]}$ Since the methods by which these two versions of guidelines were formulated were far apart, and the study results from Chinese RA patients were not included, these guidelines would not satisfy the current needs of RA diagnosis and treatment. More importantly, many new drugs were developed and have been used in the treatment of RA. There have been numerous publications from China in the past 10 years. It would be imperative to formulate a newer version of the guidelines for the diagnosis and treatment of RA.

Under the leadership of the CRA, which was led by Dr. Xiaofegn Zeng, the current president, a guideline for the diagnosis and treatment of RA was formulated in 2018. This guideline was developed based on the standardized method. This newer version of the guidelines contemplated the real-world practice of Chinese rheumatologists and emphasized the importance of the "tight-control" strategy and the goal of "treat-to-target". The guideline not only reflects the international consensus on the diagnosis and treatment of RA, but also balances the practice and resources available in the primary care settings and tertiary referral centers. The recommendations included in the guideline cover the entire process of RA management; thus, they are highly practical and easy to follow. The guidelines provide guidance for rheumatologists and other clinicians to make early diagnosis and implement "tight-control" strategy and "treat-to-target" principle in practice; they have helped to improve the overall prognosis of Chinese RA patients.

\section{Opportunities and Challenges for the Management of RA in China}

Rheumatology is the youngest subspecialty of internal medicine in China, with less attention from health authorities and the public. This is reflected by the unbalanced development of rheumatology in the country and the serious shortage of rheumatologists. On October 31, 2019, the National Health Commission of the People's Republic of China issued "Guideline for the development and administrative management of department of rheumatology in general hospitals in China" and "The basic requirements for department of rheumatology in general hospitals in China". The goals of these guidelines and requirements were to strengthen the development and administrative management of the department of rheumatology in China and to improve the diagnosis and treatment of rheumatic diseases in China. These documents stipulate that hospitals that have not met the basic requirements must increase the number of rheumatologists, equip themselves with necessary instruments, improve the practicing environment, 
and manage the department strictly to gradually develop a standard department of rheumatology. These documents also specify that secondary hospitals must set up free-standing outpatient clinics for rheumatic diseases and must allocate a certain number of in-patient beds. These two documents are critical to the development of rheumatology and would move the development of rheumatology forward. We may say that the spring for rheumatology development in China is coming.

The Department of Rheumatology of PUMCH is the birthplace of Chinese rheumatology, as a result of the efforts of pioneers who spearheaded clinical studies on rheumatic diseases in China. In May 2019, the department was named the "National Clinical Research Center for Dermatologic and Immunologic Diseases" (NCRC-DID), the only national clinical research center for rheumatic diseases of the country. This center was jointly granted by the Ministry of Science and Technology of People's Republic of China, National Health Commissions of People's Republic of China, Logistics Support Department of Ministry of National Defense of People's Republic of China, and National Medical Products Administration. The center as well as these guidelines and requirements will speed up the development of Chinese rheumatology.

Despite the good opportunities, there are many challenges ahead. To improve the awareness of RA and further standardize the diagnosis and treatment, it is essential to improve the quality of care for RA in the country. Without the availability of high-quality healthcare, RA patients may not be able to benefit from the recent progress in the field of rheumatology. Low-levels of awareness of RA among Chinese healthcare authorities and professionals, as well as the public, would function as an obstacle for Chinese rheumatologists to make further progress or conduct highquality clinical research. Therefore, initiation of country-

Conflict of Interest

None declared.

References

[1] Hresko A, Lin TC, Solomon DH. Medical Care Costs Associated with Rheumatoid Arthritis in the US: A Systematic Literature Review and Meta-Analysis. Arthritis Care Res (Hoboken). 2018;70(10):1431-1438.

[2] Beasley RP, Bennett PH, Lin CC. Low Prevalence of Rheumatoid Arthritis in Chinese. Prevalence Survey in A Rural Community. J Rheumatol (Suppl). 1983;10:11-15. wide epidemiological studies will be an important focus of future efforts which aim to address the challenges posed by inadequate awareness-levels concerning rheumatology. This would help understand the current status and challenges of RA management in depth, build up a high-quality rheumatologist workforce, educate high-level researchers in rheumatic diseases, develop a multidisciplinary team at centers for rheumatic disease management, and explore preventive measures. Another important approach is to raise funds-including pharmaceutical donations-to support joint research on the RA pathogenesis and targeted therapy. This would speed up the development of novel therapies. Strengthening the prevention and management measures associated with RA is a pathway to change the current status of diagnosis and treatment. To do this, the network for RA research needs to be enlarged, the environment for collaboration and sharing between different parties in research and management of RA should be created, and high-quality evidence needs to be provided.

\section{Prospect for the Future Development of Chinese Rheumatology}

To meet the needs of rheumatic diseases prevention, a nation-wide research alliance has been developed recently under the support of national key research and development projects. In addition, a national network of research and collaboration has been formed, a national RA patient cohort and biobank has been developed, a series of research work has been published, and guidelines for RA diagnosis and treatment have been issued. All these efforts have facilitated advancements in the development of Chinese rheumatology. We would predict that the naming and establishment of the NCRC would become the engine and motive force for the rapid development of Chinese rheumatology.
[3] Zeng XF et al. The Systemic Review of the Studies on Disease Burden and Quality of Life of Rheumatoid Arthritis in China. Chin J Evid-Based Med. 2013;13(3):300-307.

[4] Cross M, Smith E, Hoy D, et al. The Global Burden of Rheumatoid Arthritis: Estimates from the Global Burden of Disease 2010 Study. Ann Rheum Dis. 2014;73(7):1316-1322.

[5] Myasoedova E, Davis J, Matteson EL, Crowson CS. Is the 
epidemiology of rheumatoid arthritis changing? Results from a population-based incidence study, 1985-2014. Ann Rheum Dis. 2020;79(4):440-444.

[6] Zhou Y, Wang X, An Y, et al. A Multi-Center Survey on the Disability and Functional Impairment of Rheumatoid Arthritis Patients in China. Chin J Rheumatol. 2013;17(8):526-532.

[7] Carmona L, Cross M, Williams B,et al. Rheumatoid Arthritis. Best Pract Res Clin Rheumatol. 2010;24(6):733-745.

[8] DALYs GBD, HALE Collaborators. Global, Regional, and National Disability-Adjusted Life-Years (DALYs) for 359 Diseases and Injuries and Healthy Life Expectancy (HALE) for 195 Countries and Territories, 1990-2017: A Systematic Analysis for the Global Burden of Disease Study 2017. Lancet. 2018;392(10159):1859-1922.

[9] Liu L, Mao Y, Gao L. The Analysis of the Global Drug Development for Rheumatoid Arthritis. Sci Technol Rev. 2016;34(24):44-55.

[10] Guelfucci F, Kaneko Y, Mahlich J, et al. Cost of Depression in Japanese Patients with Rheumatoid Arthritis: Evidence from Administrative Data. Rheumatol Ther. 2018;5(1):171-183.

[11] Shi F, Gu K, Lu W, et al. Disease Burden Analysis Patients with Arthritis is in Shanghai. Chin Heal Res. 2007;10(2):92-94.

[12] Gu S, Deng Q, Pan X, et al. Survey on the Awareness of Rheumatoid Arthritis in Part of the Citizen in Langzhong City. Health Guide. 2017;30:100,72.

[13] Liu X, Jia Y, An Y, et al. Analysis of the Current Situation of Medical Care Seeking and Treatment of Chinese Rheumatoid Arthritis Patients. Chin J Rheumatol. 2008;12(9):637-639.

[14] Liu N, Wang XY, Wang XR, et al. Survey on the Use of Glucocorticosteroid in Rheumatoid Arthritis Patients in MultiCenters of China. Chin Med. 2016;11(8):1216-1221.

[15] Jin S, Li M, Fang Y,et al. Chinese Registry of Rheumatoid Arthritis (CREDIT): II. Prevalence and Risk Factors of Major Comorbidities in Chinese Patients with Rheumatoid Arthritis. Arthritis Res Ther. 2017;19(1):251.

[16] Wu B, Wilson A, Wang FF, et al. Cost Effectiveness of Different Treatment Strategies in the Treatment of Patients with Moderate to Severe Rheumatoid Arthritis in China. PLoS One. 2012;7(10):e47373.

[17] Chinese Rheumatology Association. Report on the Survey on the Current Status of Chinese Rheumatologists. 2009;13(6):407-409.

[18] Song G, Feng T, Zhao R, et al. CD109 Regulates the Inflammatory Response and is Required for the Pathogenesis of Rheumatoid Arthritis. Ann Rheum Dis. 2019;78(12):16321641.

[19] Zhu H, Wu LF, Mo XB, et al. Rheumatoid Arthritis-Associated DNA Methylation Sites in Peripheral Blood Mononuclear Cells. Ann Rheum Dis. 2019;78(1):36-42.

[20] Sun W, Meednu N, Rosenberg A, et al. B cells Inhibit Bone Formation in Rheumatoid Arthritis by Suppressing Osteoblast Differentiation. Nat Commun. 2018;9(1):5127.

[21] Feng $\mathrm{H}$, Zhang Z, Meng Q, et al. Rapid Response Fluorescence Probe Enabled In Vivo Diagnosis and Assessing Treatment Response of Hypochlorous Acid-Mediated Rheumatoid Arthritis. Adv Sci (Weinh). 2018;5(8):1800397.

[22] Wang D, Zhang J, Lau J, et al. Mechanisms of Lung Disease Development in Rheumatoid Arthritis. Nat Rev Rheumatol. 2019;15(10):581-596.

[23] Pan H, Guo R, Ju Y, et al. A Single Bacterium Restores the Microbiome Dysbiosis to Protect Bones from Destruction in A Rat Model of Rheumatoid Arthritis. Microbiome. 2019;7(1):107.

[24] Huang CC, Chiou CH, Liu SC, et al. Melatonin Attenuates TNF-Alpha and IL-1beta Expression in Synovial Fibroblasts and Diminishes Cartilage Degradation: Implications for the Treatment of Rheumatoid Arthritis. J Pineal Res. 2019;66(3):12560.

[25] Gao F, Yuan Q, Cai P, et al. Au Clusters Treat Rheumatoid Arthritis with Uniquely Reversing Cartilage/Bone Destruction. Adv Sci (Weinh). 2019;6(7):1801671.

[26] Yamanaka H, Tanaka E, Nakajima A, et al. A Large Observational Cohort Study of Rheumatoid Arthritis, IORRA: Providing Context for Today's Treatment Options. Mod Rheumatol. 2019;1-6.

[27] Chinese Rheumatology Rheumatology. The Guideline for the Diagnosis and Treatment of Rheumatoid Arthritis (Draft). Chin J Rheumatol. 2003;7(4):250-254.

[28] Tian X, Wang Q, Li M, et al. 2018 Chinese Guidelines for the Diagnosis and Treatment of Rheumatoid Arthritis. Rhuematol Immunol Res. 2021;1(2):1-14. 